\title{
Hamiltonian formalism of the Landau-Lifschitz equation for a spin chain with full anisotropy
}

\author{
Nian-Ning Huang, Hao Cai $*$ Tian Yan, and Fan-Rong Xu \\ Department of Physics, Wuhan University, Wuhan 430072, P.R.China
}

(Dated: November 16, 2018)

\begin{abstract}
The Hamiltonian formalism of the Landau-Lifschitz equation for a spin chain with full anisotropy is formulated completely, which constructs a stable base for further investigations.
\end{abstract}

\section{INTRODUCTION}

The Landau-Lifschitz(L-L) equation for a spin chain with full anisotropy is one of the most important completely integrable nonlinear evolution equations ${ }^{1}$. The compatibility pair were first given by E.K.Sklyanin ${ }^{2}$ in terms of elliptic functions. The Hamiltonian formalism was also examined, but the reduction procedure and results have some open points so that the final results are not conclusive $e^{2}$. On the other hand, there were some works trying to solve the equation based upon these compatibility pair, but exact solutions were not found until now ${ }^{3.4}$. Because these problems are more basic, it is worth to putting a lot of work. In this paper the Hamiltonian formalism is formulated to provide a base for further investigations.

\section{LANDAU-LIFSCHITZ EQUATION}

The L-L equation for anisotropic spin chain is in the form of

$$
S_{t}=S \times S_{x x}+S \times J S, \quad|S|=1
$$

in which $J=\operatorname{diag}\left(J_{1}, J_{2}, J_{3}\right), J_{1} \leq J_{2} \leq J_{3}$, describing nonlinear spin waves propagating in a direction orthogonal to the anisotropic axis, and the suffices $x$ and $t$ denote the corresponding partial derivatives. Though spin is a quantum quantity satisfying quantum bracket relation, but if one introduces Lie-Poisson bracket for classical spin, all the procedure of disposal is the same as the usual classical one $\mathrm{I}^{\underline{1}}$. The Lie-Poisson bracket is given by

$$
\left\{S_{a}(x), S_{b}(y)\right\}=-\epsilon_{a b c} S_{c}(x) \delta(x-y)
$$

where $\epsilon_{a b c}$ is a fully anti-symmetric tensor, $a, b, c=1,2,3$. By using it we write eq. (11) in the form of the canonical equation

$$
\partial_{t} S_{a}(x)=\left\{S_{a}(x), H\right\}
$$

in which the Hamiltonian is

$$
H=\int_{-\infty}^{\infty} d x \mathcal{H}(x), \quad \mathcal{H}(x)=\frac{1}{2} \sum_{a}\left[\left(\partial_{x} S_{a}(x)\right)^{2}-J_{a} S_{a}(x)^{2}\right]
$$

\section{JOST SOLUTIONS}

The compatibility pair of the equation are a couple of $2 \times 2$ matrices given by E.K.Sklyanin ${ }^{2}$,

$$
L=-i \sum_{a} \omega_{a}(\lambda) S_{a} \sigma_{a}
$$

and

$$
M=-i \sum_{a, b, c} \omega_{a}(\lambda) S_{b} S_{c x} \sigma_{a} \epsilon_{a b c}+i \sum_{a, b, c} \omega_{b}(\lambda) \omega_{c}(\lambda) S_{a} \sigma_{a}\left|\epsilon_{a b c}\right|
$$

in which

$$
\omega_{1}(\lambda)=\rho \frac{1}{\operatorname{sn}(\lambda, \kappa)}, \quad \omega_{2}(\lambda)=\rho \frac{\operatorname{dn}(\lambda, \kappa)}{\operatorname{sn}(\lambda, \kappa)}, \quad \omega_{3}(\lambda)=\rho \frac{\operatorname{cn}(\lambda, \kappa)}{\operatorname{sn}(\lambda, \kappa)}
$$


and $\operatorname{sn}(\lambda, \kappa)$, etc., are elliptic functions with modulus $\kappa$ :

$$
\kappa=\sqrt{\frac{J_{2}-J_{1}}{J_{3}-J_{1}}}, \quad \rho=\frac{1}{2} \sqrt{J_{3}-J_{1}}
$$

Since the coefficients $\omega_{a}(\lambda)$ are double-periodic functions of the parameter $\lambda$ :

$$
\omega_{a}\left(\lambda+4 m K+i 4 n K^{\prime}\right)=\omega_{a}(\lambda)
$$

where $n, m$ are integers, $K$ and $K^{\prime}$ are quarter-periods. It is sufficient to consider $\lambda$ in the fundamental period parallelogram $\Omega$ :

$$
\Omega:|\operatorname{Re} \lambda|<2 K, \quad|\operatorname{Im} \lambda|<2 K^{\prime}
$$

The first compatibility equation is now written as

$$
\partial_{x} F(x, \lambda)=L(x, \lambda) F(x, \lambda)
$$

In the limit of $|x| \rightarrow \pm \infty, S \rightarrow(0,0,1)$, and the asymptotic solution corresponding is $E(x, \lambda)=e^{-i \omega_{3} x \sigma_{3}}$. The Jost solutions of eq.(11) are then defined by

$$
\begin{array}{ll}
\Psi(x, \lambda)=(\tilde{\psi}(x, \lambda), \psi(x, \lambda)) \rightarrow e^{-i \omega_{3} x \sigma_{3}}, & \text { as } x \rightarrow \infty \\
\Phi(x, \lambda)=(\phi(x, \lambda), \tilde{\phi}(x, \lambda)) \rightarrow e^{-i \omega_{3} x \sigma_{3}}, & \text { as } x \rightarrow-\infty
\end{array}
$$

The monodromy matrix $T(\lambda)$ is given by

$$
\Phi(x, \lambda)=\Psi(x, \lambda) T(\lambda), \quad T(\lambda)=\left(\begin{array}{rr}
a(\lambda) & -\tilde{b}(\lambda) \\
b(\lambda) & \tilde{a}(\lambda)
\end{array}\right)
$$

Furthermore, from the periodic properties of $\operatorname{sn}(\lambda)$ and $\operatorname{sn}(\bar{\lambda})=\overline{\operatorname{sn}(\lambda)}$, etc., the compatibility pair (5) and (6) have the following reduction transformation properties:

$$
\begin{gathered}
L(\lambda+2 K)=\sigma_{3} L(\lambda) \sigma_{3}, \quad M(\lambda+2 K)=\sigma_{3} M(\lambda) \sigma_{3} \\
\overline{L\left(\bar{\lambda}+i 2 K^{\prime}\right)}=\sigma_{3} L(\lambda) \sigma_{3}, \quad \overline{M\left(\bar{\lambda}+i 2 K^{\prime}\right)}=\sigma_{3} M(\lambda) \sigma_{3}
\end{gathered}
$$

\section{LIE-POISSON BRACKET}

From the first compatibility equation, it is found that

$$
\frac{\delta T(\lambda)}{\delta S_{a}(z)}=\Psi^{-1}(z, \lambda)\left(i \omega_{a} \sigma_{a}\right) \Phi(z, \lambda)
$$

and

$$
\frac{\delta T^{-1}(\lambda)}{\delta S_{b}(z)}=-\Phi^{-1}(z, \lambda)\left(i \omega_{b} \sigma_{b}\right) \Psi(z, \lambda)
$$

Defining $^{5}$

$$
\left\{T(\lambda){ }^{\otimes} T^{-1}\left(\lambda^{\prime}\right)\right\}_{i k, j l}=\left\{T(\lambda)_{i j}, T^{-1}\left(\lambda^{\prime}\right)_{k l}\right\}
$$

the Lie-Poisson bracket of the monodromy matrix is now simply given in the form

$$
\left\{T(\lambda) \stackrel{\otimes}{,} T^{-1}\left(\lambda^{\prime}\right)\right\}=-\epsilon_{a b c} \int d x \frac{\delta T(\lambda)}{\delta S_{a}(x)} \otimes \frac{\delta T^{-1}\left(\lambda^{\prime}\right)}{\delta S_{b}(x)} S_{c}(x)
$$


in which the symbol $\otimes$ in the right hand side is the usual direct product. After substituting eqs.(16) and (17), the explicit expression of eq.(19) is

$$
\left\{T(\lambda) \stackrel{\otimes}{,} T^{-1}\left(\lambda^{\prime}\right)\right\}=\int d x \Psi^{-1}(x, \lambda) \Phi^{-1}\left(x, \lambda^{\prime}\right) R \Phi(x, \lambda) \Psi\left(x, \lambda^{\prime}\right)
$$

where

$$
\begin{aligned}
R=S_{3} & \left\{i \omega_{1} \omega_{2}^{\prime} \sigma_{1} \otimes i \sigma_{2}-i \omega_{2} \omega_{1}^{\prime} i \sigma_{2} \otimes \sigma_{1}\right\}+S_{1}\left\{i \omega_{2} \omega_{3}^{\prime} i \sigma_{2} \otimes \sigma_{3}-i \omega_{3} \omega_{2}^{\prime} \sigma_{3} \otimes i \sigma_{2}\right\} \\
& +S_{2}\left\{-\omega_{3} \omega_{1}^{\prime} \sigma_{3} \otimes \sigma_{1}+\omega_{1} \omega_{3}^{\prime} \sigma_{1} \otimes \sigma_{3}\right\}
\end{aligned}
$$

The eq.(20) has a simple result if the integrand in the right hand side is a full derivative of some function with respect to $x$. In order to do so, we take into account of

$$
\partial_{x}\left(\Psi^{-1}(x, \lambda) \sigma_{\alpha} \Psi\left(x, \lambda^{\prime}\right) \otimes^{\prime} \Phi^{-1}\left(x, \lambda^{\prime}\right) \sigma_{\alpha} \Phi(x, \lambda)\right)
$$

where $\sigma_{0}=I$, and $\sigma_{a}$ is Pouli's matrix for $a=1,2,3$. Here another type of direct product is introduced

$$
A_{i m} B_{l j}=\left(A \otimes^{\prime} B\right)_{i l, j m}
$$

Using eqs.(5) and (11), it is obvious that

$$
\Psi^{-1}(x, \lambda) \Phi^{-1}\left(x, \lambda^{\prime}\right) W_{\alpha} \Phi(x, \lambda) \Psi\left(x, \lambda^{\prime}\right)
$$

in which

$$
\begin{gathered}
W_{0}=i\left(\omega_{a}-\omega_{a}^{\prime}\right) S_{a}\left(\sigma_{a} \otimes^{\prime} I-I \otimes^{\prime} \sigma_{a}\right) \\
W_{a}=i S_{b}\left(\omega_{b} \sigma_{b} \sigma_{a}+\omega_{b}^{\prime} \sigma_{a} \sigma_{b}\right) \otimes^{\prime} \sigma_{a}+i S_{b} \sigma_{a} \otimes^{\prime}\left(\omega_{b}^{\prime} \sigma_{b} \sigma_{a}+\omega_{b} \sigma_{a} \sigma_{b}\right)
\end{gathered}
$$

In fact, eq.(20) can be expressed as a linear combination of terms in eq.(22), since $R$ in eq. (21) is expressed as a linear combination of $W_{\alpha}$ in eqs.(25) and (26):

$$
R=f_{0} W_{0}+f_{3} W_{3}+f_{1} W_{1}+f_{2} W_{2}
$$

Writing $R$ and $W_{\alpha}$ in bigger matrices, e.g. $4 \times 4$-matrices, and comparing the corresponding matrix elements, a group of equations for $f_{\alpha}$ are given in the Appendix A.

Eq.(20) is then

$$
\left\{T(\lambda) \stackrel{\otimes}{,} T^{-1}\left(\lambda^{\prime}\right)\right\}=\sum_{\alpha} f_{\alpha} \Delta_{\alpha}
$$

where

$$
\left.\Delta_{\alpha} \equiv \Psi^{-1}(x, \lambda) \sigma_{\alpha} \Psi\left(x, \lambda^{\prime}\right) \otimes^{\prime} \Phi^{-1}\left(x, \lambda^{\prime}\right) \sigma_{\alpha} \Phi(x, \lambda)\right|_{x=-L} ^{x=L}
$$

\section{EXPLICIT EXPRESSION OF LIE-POISSON BRACKET}

On the other hand, we have

$$
\sum_{\alpha=0}^{3} f_{\alpha} \Delta_{\alpha}=f_{0}\left(\Delta_{0}+\Delta_{3}+\Delta_{1}+\Delta_{2}\right)+\left(f_{3}-f_{0}\right) \Delta_{3}+\left(f_{1}-f_{0}\right) \Delta_{1}+\left(f_{2}-f_{0}\right) \Delta_{2}
$$

Since $\left\{T(\lambda){ }^{\otimes} T^{-1}\left(\lambda^{\prime}\right)\right\}$ is definite and only the differences $f_{3}-f_{0}, f_{1}-f_{0}, f_{2}-f_{0}$ can be determined from eqs. A4 $\sim$ (A6), we should see that $\Delta_{0}+\Delta_{3}+\Delta_{1}+\Delta_{2}=0$, which means that the value of $f_{0}$ is of no importance and may be assumed to be 0 . Thus we have

$$
\left\{T(\lambda) \stackrel{\otimes}{,} T^{-1}\left(\lambda^{\prime}\right)\right\}=f_{3}(\Delta(b)-\Delta(b 0))+f_{1}(-\Delta(b)+\Delta(b 1))+f_{2}(-\Delta(b)-\Delta(b 1))
$$


in which

$$
f_{3}=\frac{\omega_{1} \omega_{2}^{\prime}+\omega_{2} \omega_{1}^{\prime}}{2\left(\omega_{3}-\omega_{3}^{\prime}\right)}, \quad f_{1}=\frac{\omega_{2} \omega_{3}^{\prime}+\omega_{3} \omega_{2}^{\prime}}{2\left(\omega_{1}-\omega_{1}^{\prime}\right)}, \quad f_{2}=\frac{\omega_{3} \omega_{1}^{\prime}+\omega_{1} \omega_{3}^{\prime}}{2\left(\omega_{2}-\omega_{2}^{\prime}\right)}
$$

and the explicit expressions of $\Delta(b), \Delta(b 0)$ and $\Delta(b 1)$ are given in the Appendix B.

Because of properties shown in (14), $\lambda$ can be restricted in the region $\Omega_{+}$:

$$
\Omega_{+}: 0<\operatorname{Re} \lambda<2 K, \quad 0<\operatorname{Im} \lambda<2 K^{\prime}
$$

In this restriction, $\Delta(b 1)$ has no contribution, and (31) reduces to

$$
\begin{aligned}
& \left\{T(\lambda){ }^{\otimes} T^{-1}\left(\lambda^{\prime}\right)\right\}=f_{3}(\Delta(b)-\Delta(b 0)) \\
& \quad=-\frac{\omega_{1} \omega_{2}^{\prime}+\omega_{2} \omega_{1}^{\prime}}{2}\left(\begin{array}{cccc}
0 & -\frac{1}{\omega_{3}-\omega_{3}^{\prime}+i 0} a \tilde{b}^{\prime} & -\frac{1}{\omega_{3}-\omega_{3}^{\prime}+i 0} \tilde{a}^{\prime} \tilde{b} & 0 \\
-\frac{1}{\omega_{3}-\omega_{3}^{\prime}+i 0} b^{\prime} a & 0 & i 2 \pi \delta\left(\omega_{3}-\omega_{3}^{\prime}\right)|a|^{2} & \frac{1}{\omega_{3}-\omega_{3}^{\prime}-i 0} \tilde{b} a^{\prime} \\
-\frac{1}{\omega_{3}-\omega_{3}^{\prime}+i 0} b \tilde{a}^{\prime} & -i 2 \pi \delta\left(\omega_{3}-\omega_{3}^{\prime}\right)|a|^{2} & 0 & \frac{1}{\omega_{3}-\omega_{3}^{\prime}-i 0} \tilde{b}^{\prime} \tilde{a} \\
0 & \frac{1}{\omega_{3}-\omega_{3}^{\prime}-i 0} a^{\prime} b & \frac{1}{\omega_{3}-\omega_{3}^{\prime}-i 0} \tilde{a} b^{\prime} & 0
\end{array}\right)
\end{aligned}
$$

where $\omega_{j}, \omega_{j}^{\prime}$ mean $\omega_{j}(\lambda), \omega_{j}\left(\lambda^{\prime}\right)$.

From eq.(34), there are

$$
\begin{aligned}
\left\{a(\lambda), b\left(\lambda^{\prime}\right)\right\} & =\frac{\omega_{1} \omega_{2}^{\prime}+\omega_{2} \omega_{1}^{\prime}}{2} \frac{1}{\omega_{3}-\omega_{3}^{\prime}+i 0} a b^{\prime} \\
& =\frac{\omega_{1} \omega_{2}^{\prime}+\omega_{2} \omega_{1}^{\prime}}{2\left(\omega-\omega^{\prime}\right)} a b^{\prime}-\frac{\omega_{1} \omega_{2}^{\prime}+\omega_{2} \omega_{1}^{\prime}}{2} i \pi \delta\left(\omega_{3}-\omega_{3}^{\prime}\right) a b^{\prime} \\
\left\{\tilde{a}(\lambda), b\left(\lambda^{\prime}\right)\right\} & =-\frac{\omega_{1} \omega_{2}^{\prime}+\omega_{2} \omega_{1}^{\prime}}{2} \frac{1}{\omega_{3}-\omega_{3}^{\prime}-i 0} \tilde{a} b^{\prime} \\
& =-\frac{\omega_{1} \omega_{2}^{\prime}+\omega_{2} \omega_{1}^{\prime}}{2\left(\omega-\omega^{\prime}\right)} a b^{\prime}-\frac{\omega_{1} \omega_{2}^{\prime}+\omega_{2} \omega_{1}^{\prime}}{2} i \pi \delta\left(\omega_{3}-\omega_{3}^{\prime}\right) a b^{\prime}
\end{aligned}
$$

and then

$$
\left\{|a(\lambda)|^{2}, b\left(\lambda^{\prime}\right)\right\}=-i 2 \pi \delta\left(\omega_{3}-\omega_{3}^{\prime}\right) \omega_{1} \omega_{2}|a|^{2} b^{\prime}
$$

Furthermore, in the restriction $\Omega_{+}$, there are

$$
\delta\left(\omega_{3}-\omega_{3}^{\prime}\right)=\frac{1}{\frac{d \omega_{3}(\lambda)}{d \lambda}} \delta\left(\lambda-\lambda^{\prime}\right)
$$

as $\lambda=\lambda^{\prime}$, and

$$
\frac{\omega_{1} \omega_{2}^{\prime}+\omega_{2} \omega_{1}^{\prime}}{2}=\rho^{2} \frac{\operatorname{dn}(\lambda)}{\operatorname{sn}^{2}(\lambda)}=-\rho \frac{d \omega_{3}(\lambda)}{d \lambda}
$$

As a result, eqs.(35), (36) and (37) become

$$
\begin{gathered}
\left\{a(\lambda), b\left(\lambda^{\prime}\right)\right\}=\frac{\omega_{1} \omega_{2}^{\prime}+\omega_{2} \omega_{1}^{\prime}}{2\left(\omega-\omega^{\prime}\right)} a(\lambda) b\left(\lambda^{\prime}\right)+i \pi \rho \delta\left(\lambda-\lambda^{\prime}\right) a(\lambda) b\left(\lambda^{\prime}\right) \\
\left\{\tilde{a}(\lambda), b\left(\lambda^{\prime}\right)\right\}=-\frac{\omega_{1} \omega_{2}^{\prime}+\omega_{2} \omega_{1}^{\prime}}{2\left(\omega-\omega^{\prime}\right)} \tilde{a}(\lambda) b\left(\lambda^{\prime}\right)+i \pi \rho \delta\left(\lambda-\lambda^{\prime}\right) \tilde{a}(\lambda) b\left(\lambda^{\prime}\right)
\end{gathered}
$$

and

$$
\left\{|a(\lambda)|^{2}, b\left(\lambda^{\prime}\right)\right\}=i 2 \pi \rho \delta\left(\lambda-\lambda^{\prime}\right)|a(\lambda)|^{2} b\left(\lambda^{\prime}\right)
$$




\section{ACTION-ANGLE VARIABLES IN CONTINUOUS SPECTRUM}

As known in inverse scattering transform, $a(\lambda)$ and $\tilde{a}(\lambda)$ are independent of $t$, and the phase of $b(\lambda)$ and $\tilde{b}(\lambda)$ is a function of $t$, which is determined by the asymptotic form of $M$ in eq. (6). We have

$$
b(t, \lambda)=b(0, \lambda) e^{-i 4 \omega_{1} \omega_{2} t}
$$

and then the angle variable is defined as

$$
Q(\lambda)=\arg b(\lambda)=\frac{1}{i} \ln b(\lambda)
$$

The action variable $P(\lambda)$ is a function of $a(\lambda)$ and $\tilde{a}(\lambda)$, and usually assumed to be

$$
P(\lambda)=F\left(|a(\lambda)|^{2}\right)
$$

where $F$ is an unknown function. As these two variables are canonical variables, it should be

$$
\left\{P(\lambda), Q\left(\lambda^{\prime}\right)\right\}=-\delta\left(\lambda-\lambda^{\prime}\right)
$$

By eq.(42), we find

$$
\left\{F\left(|a(\lambda)|^{2}\right), Q\left(\lambda^{\prime}\right)\right\}=F^{\prime}\left(|a(\lambda)|^{2}\right) 2 \pi \rho \delta\left(\lambda-\lambda^{\prime}\right)|a(\lambda)|^{2}
$$

where $F^{\prime}$ is derivative of $F$ with respect of its argument. Comparing it with eq.(46), we obtain

$$
F^{\prime}\left(|a(\lambda)|^{2}\right) 2 \pi \rho|a(\lambda)|^{2}=-1
$$

and thus

$$
P(\lambda)=F\left(|a(\lambda)|^{2}\right)=-\frac{1}{2 \pi \rho} \ln |a(\lambda)|^{2}
$$

Eq.(44) yields

$$
Q(\lambda, t)=Q(\lambda, 0)-4 \omega_{1} \omega_{2}
$$

Hence the Hamiltonian is

$$
H=\int_{0}^{2 K} d \lambda 4 \omega_{1} \omega_{2} P(\lambda)=-\frac{2}{\pi} \int_{0}^{2 K} d \lambda \frac{\rho \operatorname{dn}(\lambda)}{\operatorname{sn}^{2}(\lambda)} \ln |a(\lambda)|^{2}
$$

Therefore, the Hamiltonian has two kinds of expressions: one is an integral with respect to $x$ in eq.(4), and the other is an integral with respect to the spectral parameter in eq.(51). Now it is necessary to derive a conservative quantity which has two integral forms compatible with the Hamiltonian.

\section{CONSERVATIVE QUANTITIES}

In the inverse scattering transform, the conservative quantities are derived from the asymptotic form of the first compatibility equation. In the limit $|\lambda| \rightarrow 0$ or $|k| \rightarrow \infty\left(k=\rho \lambda^{-1}\right)$, by using the asymptotic expansion of elliptic functions, we have

$$
L \rightarrow-i\left(k+k^{-1} r_{a}\right) S_{a} \sigma_{a}+\cdots
$$

and

$$
\left\{r_{1}, r_{2}, r_{3}\right\}=4 \rho^{2} \frac{1}{6}\left\{\left(1+\kappa^{2}\right),\left(1-2 \kappa^{2}\right),\left(-2+\kappa^{2}\right)\right\}
$$

From the first compatibility equation in this limit, writing

$$
\tilde{\psi}_{2}(x, k)=e^{i k x+g}
$$


and expanding

$$
g_{x}=\eta_{0}+(i 2 k)^{-1} \eta_{1}+(i 2 k)^{-2} \eta_{2}+\ldots
$$

we obtain

$$
\eta_{0}=S_{3 x}+\frac{\left(-i S_{1}+S_{2}\right)_{x}}{-i S_{1}+S_{2}}\left(1-S_{3}\right)
$$

and

$$
-2 \eta_{1}=\eta_{0 x}+2 \eta_{0}^{2}-\frac{\left(-i S_{1}+S_{2}\right)_{x}}{-i S_{1}+S_{2}}\left(\eta_{0}\right)+2 r_{a} S_{a}^{2}
$$

etc $\underline{\underline{6}}$. In general, $\eta_{0} \neq 0$. This situation appears also in the case of isotropic spin chain. In that case, an additional phase in the transmission coefficient $a(k)$ was introduced 5 to cancel the non-vanishing $\eta_{0}$. However, Takhtajan and Zakharov pointed out that this is unreasonable ${ }^{7}$. Any way, $\eta_{1}$ in eq.(57) does not give an expression compatible with the Hamiltonian in eq.(4).

It was formerly shown that the gauge equivalence between the isotropic spin chain and the nonlinear Schrödinger equation, which means, by choosing a suitable gauge, the gauge-transformed compatibility pair of isotropic spin chain has the same form of that of NLS equation. Therefore, the conservative quantities for the isotropic spin chain can be derived from those for the NLS equation by revised gauge transformation and all results expected are naturally found ${ }^{7}$.

After Takhtajan and Zakharov $\underline{\underline{7}}$, it was tried to find some equations that are gauge-equivalent to the L-L equations for a spin chain with axial symmetry. However, such equations seem not existent. From a careful analysis of the gauge equivalence between the isotropic spin chain and the NLS equation, only the leading terms of the first one of compatibility pair are essentials, while other terms corresponding and the second one of compatibility pair are of no importance. That is, a gauge is chosen such that it turns the spin in the first order of spectral parameter of the first one of compatibility pair into the 3 - axis in the spin space $\underline{\underline{6}}$.

The explicit expression of the gauge $B$ was given(see Ref.[6]). After the gauge transformation, eq.(52) turns to

$$
L^{\prime}=-i\left(k+2 r_{a} S_{a}^{2}\right) \sigma_{3}+U+k^{-1} V+\cdots
$$

where $U \equiv B_{x} B^{-1}=\left(\begin{array}{cc}0 & u \\ -\bar{u} & 0\end{array}\right)$ and $V$ is an $2 \times 2$ matrix with vanishing diagonal elements.

Replacing $L$ by $L^{\prime}$, the similar procedure for eq.(56) and (57) yields

$$
\eta_{0}^{\prime}=0, \quad \eta_{1}^{\prime}=-|u|^{2}+2\left(r_{1} S_{1}^{2}+r_{2} S_{2}^{2}+r_{3} S_{3}^{2}\right)
$$

Noticing (8), there are

$$
\left\{r_{1}, r_{2}, r_{3}\right\}=\frac{1}{6}\left\{J_{3}+J_{2}-2 J_{1}, J_{3}-2 J_{2}+J_{1},-2 J_{3}+J_{1}+J_{2}\right\} \sim-\frac{1}{2}\left\{J_{1}, J_{2}, J_{3}\right\}
$$

since common constant is immateriale ${ }^{8}$. As shown in the case of isotropic spin chain ${ }^{7}$

$$
4|u|^{2}=-S_{a x} S_{a x}
$$

We finally obtain

$$
\eta_{1}^{\prime}=\frac{1}{2}\left(S_{a x} S_{a x}-\left(J_{1} S_{1}^{2}+J_{2} S_{2}^{2}+J_{3} S_{3}^{2}\right)\right)
$$

which is just the density of the Hamiltonian in eq.(4).

\section{EXPRESSION OF $a(\lambda)$}

Eq.(59) indicates $a(k) \rightarrow 0$ as $k \rightarrow \infty$, so that in this domain

$$
\ln a(k)=\frac{1}{i \pi} \int d k^{\prime} \frac{\ln \left|a\left(k^{\prime}\right)\right|^{2}}{k^{\prime}-k}
$$


We have

$$
\begin{gathered}
\ln a(k)=I_{0}+I_{1}(i 2 k)^{-1}+\cdots \\
I_{0}=0, \quad I_{1}=-\frac{2}{\pi} \int d k^{\prime} \ln \left|a\left(k^{\prime}\right)\right|^{2}
\end{gathered}
$$

In terms of $\lambda\left(k=2 \rho \lambda^{-1}\right)$, eq. 63 is re-written in the domain of $\lambda \approx 0$

$$
\ln a(\lambda)=\frac{1}{i \pi} \int d \lambda^{\prime} \frac{\ln \left|a\left(\lambda^{\prime}\right)\right|^{2}}{\left(\lambda^{\prime}-\lambda\right)} \frac{\lambda}{\lambda^{\prime}}
$$

so that

$$
I_{1}=-\frac{2}{\pi} \int d \lambda^{\prime} \frac{\rho}{\lambda^{\prime 2}} \ln \left|a\left(\lambda^{\prime}\right)\right|^{2}
$$

It is equal to eq. (51) in this domain, since $\operatorname{dn}(\lambda) \approx 1$ and $\operatorname{sn}(\lambda) \approx \lambda^{-1}$.

Since the general formula in this case is invariant with double-periodic transformation, eq. 66 may be rewritten as

$$
\ln a\left(\omega_{3}(\lambda)\right)=\frac{1}{i \pi} \int_{\Omega_{+}} d \omega_{3}\left(\lambda^{\prime}\right) \frac{\ln \left|a\left(\omega_{3}\left(\lambda^{\prime}\right)\right)\right|^{2}}{\omega_{3}\left(\lambda^{\prime}\right)-\omega_{3}(\lambda)}
$$

where the integral domain is real axis in $\Omega_{+}$given in eq.(33). We obtain

$$
\ln a\left(\omega_{3}(\lambda)\right)=I_{0}-i I_{1} \frac{\lambda}{2 \rho}+\cdots
$$

where $I_{0}=0$ and

$$
I_{1}=\frac{2}{\pi} \int_{\Omega_{+}} d \omega_{3}\left(\lambda^{\prime}\right) \ln \left|a\left(\omega_{3}\left(\lambda^{\prime}\right)\right)\right|^{2}=\frac{2}{\pi} \int_{\Omega_{+}} d \lambda^{\prime} \frac{d \omega_{3}}{d \lambda^{\prime}} \ln \left|a\left(\omega_{3}\left(\lambda^{\prime}\right)\right)\right|^{2}
$$

It approaches to eq. (51) in the domain of $|\lambda| \approx 0$.

\section{DISCRETE SPECTRUM}

Eq.(59) should include the discrete part

$$
a_{d}(k)=\prod_{j} \frac{k-k_{j}}{k-\bar{k}_{j}}
$$

where $k_{j}$ in the complex $k$-plane. It can be transformed into $\lambda$-plane, that is

$$
a_{d}(\lambda)=\prod_{j} \frac{\lambda-\lambda_{j}}{\lambda-\bar{\lambda}_{j}} \frac{\bar{\lambda}_{j}}{\lambda_{j}}
$$

In general, we write

$$
a_{d}\left(\omega_{3}(\lambda)\right)=\prod_{j} \frac{\omega_{3}(\lambda)-\omega_{3}\left(\lambda_{j}\right)}{\omega_{3}(\lambda)-\overline{\omega_{3}\left(\lambda_{j}\right)}}
$$

In the limit of $|\lambda| \approx 0$, it coincides with eq.(172) since $\omega_{3}(\lambda) \rightarrow \rho \lambda^{-1}$ in the limit of $|\lambda| \rightarrow 0$.

Extending analytically into $\Omega_{+}$, the left hand side of eq.(35) is

$$
\begin{aligned}
& \left\{\ln a\left(\omega_{3}(\lambda)\right), b\left(\lambda_{k}\right)\right\}=\cdots+\sum_{j}\left\{\left(\ln \left(\omega_{3}(\lambda)-\omega_{3}\left(\lambda_{j}\right)\right)-\ln \left(\omega_{3}(\lambda)-\overline{\omega_{3}\left(\lambda_{j}\right)}\right)\right), b\left(\lambda_{k}\right)\right\} \\
& =\cdots-\sum_{j} \frac{1}{\omega_{3}(\lambda)-\omega_{3}\left(\lambda_{j}\right)}\left\{\omega_{3}\left(\lambda_{j}\right), b\left(\lambda_{k}\right)\right\}+\sum_{j} \frac{1}{\omega_{3}(\lambda)-\overline{\omega_{3}\left(\lambda_{j}\right)}}\left\{\overline{\omega_{3}\left(\lambda_{j}\right)}, b\left(\lambda_{k}\right)\right\}
\end{aligned}
$$


where $\lambda_{k}, \lambda_{j} \in \Omega_{+}$and the right hand side is

$$
\frac{\omega_{1}(\lambda) \omega_{2}\left(\lambda_{k}\right)+\omega_{2}\left(\lambda_{k}\right) \omega_{1}(\lambda)}{2} \frac{1}{\omega_{3}(\lambda)-\omega_{3}\left(\lambda_{k}\right)} b\left(\lambda_{k}\right)
$$

Eq. (75) has a pole at $\omega_{3}(\lambda)=\omega_{3}\left(\lambda_{k}\right)$ so that

$$
\left\{\omega_{3}\left(\lambda_{j}\right), b\left(\lambda_{k}\right)\right\}=-\frac{\omega_{1}\left(\lambda_{j}\right) \omega_{2}\left(\lambda_{k}\right)+\omega_{2}\left(\lambda_{k}\right) \omega_{1}\left(\lambda_{j}\right)}{2} b\left(\lambda_{k}\right) \delta_{j k}=\left.\rho \frac{d \omega_{3}}{d \lambda}\right|_{\lambda_{j}} b\left(\lambda_{k}\right) \delta_{j k}
$$

as seen in eq.(39). Then eq.(76) gives

$$
\left\{\lambda_{j}, b\left(\lambda_{k}\right)\right\}=b\left(\lambda_{k}\right) \delta_{j k}
$$

\section{ACTION-ANGLE VARIABLES IN DISCRETE SPECTRUM}

We introduce the action-angle variables in discrete spectrum

$$
P_{j}=F\left(\omega_{3}\left(\lambda_{j}\right)\right), \quad Q_{j}=\ln b\left(\omega_{3}\left(\lambda_{j}\right)\right)
$$

Then we find

$$
\left\{P_{j}, b\left(\lambda_{k}\right)\right\}=\frac{d F}{d \omega_{3}\left(\lambda_{j}\right)}\left\{\omega_{3}\left(\lambda_{j}\right), b\left(\lambda_{k}\right)\right\}=\frac{d F}{d \omega_{3}\left(\lambda_{j}\right)} \frac{d \omega_{3}}{d \lambda_{j}} b\left(\lambda_{k}\right) \delta_{j k}
$$

namely,

$$
\frac{d F}{d \omega_{3}\left(\lambda_{j}\right)} \frac{d \omega_{3}}{d \lambda_{j}}=1
$$

and then

$$
P\left(\omega_{3}\left(\lambda_{j}\right)\right)=\lambda_{j}
$$

From eq. (73) the discrete part of Hamiltonian is

$$
H_{d}=i 4 \rho \sum_{n}\left(-\omega_{3}\left(\lambda_{n}\right)+\overline{\omega_{3}\left(\lambda_{n}\right)}\right)
$$

Here $\lambda_{n}$ lies in $\Omega_{+}$, and the factor 4 stands for four zeros of $a(\lambda)$ in $\Omega$ for a single soliton case. We thus obtain

$$
\left\{H_{d}, b\left(\lambda_{k}\right)\right\}=i 4 \rho \frac{d \omega_{3}}{d \lambda_{j}}\left\{\lambda_{j}, b\left(\lambda_{k}\right)\right\}=-i 4 \omega_{1} \omega_{2} b\left(\lambda_{k}\right)
$$

which is just canonical equation for $b\left(\lambda_{k}\right)$.

\section{CONCLUDING REMARKS}

The Hamiltonian theory to the L-L equation for a spin chain with full anisotropy was examined by E.K.Sklyanin ${ }^{2}$. As mentioned, the conservative quantities derived by him are not compatible with the usual conservative quantities, such as the Hamiltonian, which affirmatively concludes that his procedure is unreasonable. Moreover, some other results may not be beyond doubt. For example, his eq.(2.15) written in the present notation is

$$
\left\{a(\lambda), b\left(\lambda^{\prime}\right)\right\}=-\omega_{3}\left(\lambda-\lambda^{\prime}+i 0\right) a(\lambda) b\left(\lambda^{\prime}\right)
$$

which is questionable, as comparing with eqs.(35) or (40).

Mikhailov and Rodin tried to solve the Landau-Lifschitz equation for a spin chain with full anisotropy based upon the compatibility pair given by Sklyanin. But explicit solutions were not given. In the isotropic spin case, the Jost solutions do not approach to the free Jost solutions as spectral parameter $|k| \rightarrow \infty$. To construct the equations of the inverse scattering transform by Cauchy contour integral, Takhtajan introduced a redundant factor $k^{-1}$ to ensure the integral having vanishing contribution of the integral along the big circle in complex $k$-plane when the radius reaches infinity. In the case of spin chain with full anisotropy, the behaviors of the Jost solutions do not approach to the free Jost solutions when $|\lambda| \approx 0$. Rodin and Mikhailov are unable to overcome this difficulty ${ }^{3,4}$. But in order to solve the equation, it is necessary to propose a way to do so. 


\section{Acknowledgments}

The National Natural Science Foundation of China under Grant No. 10375041 supports the project.

* Electronic address: caihao2001@hotmail.com

${ }^{1}$ L.D.Landau and E.M.Lifschitz,(1935). In: Collected Papers of Landau L.D. ed. D.ter Haar, p.101. New York, Pergamon, Gordon and Breach (1965).

2 E.K.Sklyanin, Preprint LOMI K-9-79, Liningrad (1979).

3 A.V.Mikhailov, Physica 3D,73(1981); Phys.Lett. 92A,51(1982).

${ }^{4}$ Yu.L.Rodin, Lett.Math.Phys. 7,3(1983).

5 L.D.Faddeev and L.A.Takhtajan, Hamiltonian Method in the Theory of Solitons(Springer, Berlin,1987).

6 J.C.He, L.N.Shi,H.Chen and N.N.Huang, J.Phys.A37,6311(2004).

7 V.E.Zakharov and L.A.Takhtajan, Theor.Math.Phys.38,17(1979).

8 N.N.Huang, H.Cai, F.M.Liu, L.N.Shi, Chin.Phys.Lett.21,1699(2004)

\section{APPENDIX A}

To calculate $f_{\alpha}$ in (26), the terms involving $S_{3}, S_{1}, S_{2}$ in eq.(26) are

$$
\begin{aligned}
-\left(\omega_{1} \omega_{2}^{\prime} \sigma_{1} \otimes \sigma_{2}-\omega_{2} \omega_{1}^{\prime} \sigma_{2} \otimes \sigma_{1}\right)= & \left(f_{3}-f_{0}\right) i\left(\omega_{3}-\omega_{3}^{\prime}\right)\left(I \otimes^{\prime} \sigma_{3}-\sigma_{3} \otimes^{\prime} I\right) \\
& +\left(f_{1}-f_{2}\right) i\left(\omega_{3}+\omega_{3}^{\prime}\right)\left(i \sigma_{1} \otimes^{\prime} \sigma_{2}+i \sigma_{2} \otimes^{\prime} \sigma_{1}\right) \\
-\left(\omega_{2} \omega_{3}^{\prime} \sigma_{2} \otimes \sigma_{3}-\omega_{3} \omega_{2}^{\prime} \sigma_{3} \otimes \sigma_{2}\right)= & \left(f_{1}-f_{0}\right) i\left(\omega_{1}-\omega_{1}^{\prime}\right)\left(I \otimes^{\prime} \sigma_{1}-\sigma_{1} \otimes^{\prime} I\right) \\
& +\left(f_{2}-f_{3}\right) i\left(\omega_{1}+\omega_{1}^{\prime}\right)\left(i \sigma_{2} \otimes^{\prime} \sigma_{3}+i \sigma_{3} \otimes^{\prime} \sigma_{2}\right) \\
-\left(\omega_{3} \omega_{1}^{\prime} \sigma_{3} \otimes \sigma_{1}-\omega_{1} \omega_{3}^{\prime} \sigma_{1} \otimes \sigma_{3}\right)= & \left(f_{2}-f_{0}\right) i\left(\omega_{2}-\omega_{2}^{\prime}\right)\left(I \otimes^{\prime} \sigma_{2}-\sigma_{2} \otimes^{\prime} I\right) \\
& +\left(f_{3}-f_{1}\right) i\left(\omega_{2}+\omega_{2}^{\prime}\right)\left(i \sigma_{3} \otimes^{\prime} \sigma_{1}+i \sigma_{1} \otimes^{\prime} \sigma_{3}\right)
\end{aligned}
$$

among which one equation turns to one another by simple circular permutation of $1,2,3$. Noticing the direct products in two sides are different, and writing them in $4 \times 4$ matrix form, we obtain

$$
\begin{aligned}
& \omega_{1} \omega_{2}^{\prime}-\omega_{2} \omega_{1}^{\prime}=2\left(f_{1}-f_{2}\right)\left(\omega_{3}+\omega_{3}^{\prime}\right), \quad \omega_{1} \omega_{2}^{\prime}+\omega_{2} \omega_{1}^{\prime}=2\left(f_{3}-f_{0}\right)\left(\omega_{3}-\omega_{3}^{\prime}\right) \\
& \omega_{2} \omega_{3}^{\prime}-\omega_{3} \omega_{2}^{\prime}=2\left(f_{2}-f_{3}\right)\left(\omega_{1}+\omega_{1}^{\prime}\right), \quad \omega_{2} \omega_{3}^{\prime}+\omega_{3} \omega_{2}^{\prime}=2\left(f_{1}-f_{0}\right)\left(\omega_{1}-\omega_{1}^{\prime}\right) \\
& \omega_{3} \omega_{1}^{\prime}-\omega_{1} \omega_{3}^{\prime}=2\left(f_{3}-f_{1}\right)\left(\omega_{2}+\omega_{2}^{\prime}\right), \quad \omega_{3} \omega_{1}^{\prime}+\omega_{1} \omega_{3}^{\prime}=2\left(f_{2}-f_{0}\right)\left(\omega_{2}-\omega_{2}^{\prime}\right)
\end{aligned}
$$

\section{APPENDIX B}

Denoting

$$
\begin{aligned}
& A_{\alpha}(L)=\Psi^{-1}(L, \lambda) \sigma_{\alpha} \Psi\left(L, \lambda^{\prime}\right), \quad A_{\alpha}(-L)=\Psi^{-1}(-L, \lambda) \sigma_{\alpha} \Psi\left(-L, \lambda^{\prime}\right) \\
& C_{\alpha}(L)=\Phi^{-1}\left(L, \lambda^{\prime}\right) \sigma_{\alpha} \Phi(L, \lambda), \quad C_{\alpha}(-L)=\Phi^{-1}\left(-L, \lambda^{\prime}\right) \sigma_{\alpha} \Phi(-L, \lambda)
\end{aligned}
$$

We can see

$$
\begin{aligned}
& \Delta_{0} \equiv A_{0}(L) \otimes^{\prime} C_{0}(L)-A_{0}(-L) \otimes^{\prime} C_{0}(-L)=\Delta(b)+\Delta(b 0) \\
& \Delta_{3} \equiv A_{3}(L) \otimes^{\prime} C_{3}(L)-A_{3}(-L) \otimes^{\prime} C_{3}(-L)=\Delta(b)-\Delta(b 0) \\
& \Delta_{1} \equiv A_{1}(L) \otimes^{\prime} C_{1}(L)-A_{1}(-L) \otimes^{\prime} C_{1}(-L)=-\Delta(b)+\Delta(b 1) \\
& \Delta_{2} \equiv A_{2}(L) \otimes^{\prime} C_{2}(L)-A_{2}(-L) \otimes^{\prime} C_{2}(-L)=-\Delta(b)-\Delta(b 1)
\end{aligned}
$$


where

$$
\begin{aligned}
& \Delta(b) \equiv\left(\begin{array}{cccc}
0 & -a \tilde{b}^{\prime} & -\tilde{a}^{\prime} \tilde{b} & 0 \\
-b^{\prime} a & 0 & 0 & \tilde{b} a^{\prime} \\
-b \tilde{a}^{\prime} & 0 & 0 & \tilde{b}^{\prime} \tilde{a} \\
0 & a^{\prime} b & \tilde{a} b^{\prime} & 0
\end{array}\right) \\
& \Delta(b 0) \equiv\left(\begin{array}{cccc}
C_{11,11} & \tilde{b} a^{\prime} e^{i 2\left(\omega_{3}-\omega_{3}^{\prime}\right) L} & \tilde{b}^{\prime} \tilde{a} e^{-i 2\left(\omega_{3}^{\prime}-\omega_{3}\right) L} & 0 \\
a^{\prime} b e^{-i 2\left(\omega_{3}^{\prime}-\omega_{3}\right) L} & 0 & C_{12,21} & -a \tilde{b}^{\prime} e^{-i 2\left(\omega_{3}-\omega_{3}^{\prime}\right) L} \\
\tilde{a} b^{\prime} e^{i 2\left(\omega_{3}-\omega_{3}^{\prime}\right) L} & C_{21,12} & 0 & -\tilde{a}^{\prime} \tilde{b} e^{i 2\left(\omega_{3}^{\prime}-\omega_{3}\right) L} \\
0 & -b^{\prime} a e^{i 2\left(\omega_{3}^{\prime}-\omega_{3}\right) L} & -b \tilde{a}^{\prime} e^{-i 2\left(\omega_{3}-\omega_{3}^{\prime}\right) L} & C_{22,22}
\end{array}\right) \\
& C_{11,11}=\tilde{b}^{\prime} b e^{-i 2\left(\omega_{3}^{\prime}-\omega_{3}\right) L}-\tilde{b} b^{\prime} e^{i 2\left(\omega_{3}-\omega_{3}^{\prime}\right) L} \\
& C_{12,21}=a^{\prime} \tilde{a} e^{-i 2\left(\omega_{3}^{\prime}-\omega_{3}\right) L}-a \tilde{a}^{\prime} e^{-i 2\left(\omega_{3}-\omega_{3}^{\prime}\right) L} \\
& C_{21,12}=\tilde{a}^{\prime} a e^{i 2\left(\omega_{3}^{\prime}-\omega_{3}\right) L}-\tilde{a} a^{\prime} e^{i 2\left(\omega_{3}-\omega_{3}^{\prime}\right) L} \\
& C_{22,22}=b^{\prime} \tilde{b} e^{i 2\left(\omega_{3}^{\prime}-\omega_{3}\right) L}-b \tilde{b}^{\prime} e^{-i 2\left(\omega_{3}-\omega_{3}^{\prime}\right) L}
\end{aligned}
$$

and

$$
\Delta(b 1) \equiv\left(\begin{array}{cccc}
0 & \tilde{a}^{\prime} b e^{i 2\left(\omega_{3}+\omega_{3}^{\prime}\right) L} & a b^{\prime} e^{-i 2\left(\omega_{3}+\omega_{3}^{\prime}\right) L} & C_{11,22} \\
\tilde{b} \tilde{a}^{\prime} e^{i 2\left(\omega_{3}+\omega_{3}^{\prime}\right) L} & C_{12,12} & 0 & -b^{\prime} \tilde{a} e^{i 2\left(\omega_{3}+\omega_{3}^{\prime}\right) L} \\
\tilde{b}^{\prime} a e^{-i 2\left(\omega_{3}+\omega_{3}^{\prime}\right) L} & 0 & C_{21,21} & -b a^{\prime} e^{-i 2\left(\omega_{3}+\omega_{3}^{\prime}\right) L} \\
C_{22,11} & -\tilde{a}^{\prime} \tilde{b}^{i 2\left(\omega_{3}+\omega_{3}^{\prime}\right) L} & -a^{\prime} \tilde{b} e^{-i 2\left(\omega_{3}+\omega_{3}^{\prime}\right) L} & 0
\end{array}\right)
$$

Here we show the procedure for obtaining eq. B3 as an example. Firstly, there is

$$
\begin{aligned}
A_{0}(L) \otimes^{\prime} C_{0}(L)= & \left(\begin{array}{cc}
e^{i\left(\omega_{3}-\omega_{3}^{\prime}\right) L} & 0 \\
0 & e^{-i\left(\omega_{3}-\omega_{3}^{\prime}\right) L}
\end{array}\right) \\
& \otimes^{\prime}\left(\begin{array}{cc}
\tilde{a}^{\prime} a e^{i\left(\omega_{3}^{\prime}-\omega_{3}\right) L}+\tilde{b}^{\prime} b e^{-i\left(\omega_{3}^{\prime}-\omega_{3}\right) L} & -\tilde{a}{ }^{\prime} \tilde{b} e^{i\left(\omega_{3}^{\prime}-\omega_{3}\right) L}+\tilde{b}^{\prime} \tilde{a} e^{-i\left(\omega_{3}^{\prime}-\omega_{3}\right) L} \\
-b^{\prime} a e^{i\left(\omega_{3}^{\prime}-\omega_{3}\right) L}+a^{\prime} b e^{-i\left(\omega_{3}^{\prime}-\omega_{3}\right) L} & b^{\prime} \tilde{b} e^{i\left(\omega_{3}^{\prime}-\omega_{3}\right) L}+a^{\prime} \tilde{a} e^{-i\left(\omega_{3}^{\prime}-\omega_{3}\right) L}
\end{array}\right)
\end{aligned}
$$

According to the definition of $\otimes^{\prime}$ in eq.(23), the terms involving vanishing exponent $e^{0}$ and the terms involving non-vanishing exponent $e^{ \pm i 2\left(\omega_{3}-\omega_{3}^{\prime}\right) L}$ are collected separately,

$$
\left(\begin{array}{cccc}
\tilde{a}^{\prime} a & 0 & -\tilde{a}^{\prime} \tilde{b} & 0 \\
-b^{\prime} a & 0 & b^{\prime} \tilde{b} & 0 \\
0 & \tilde{b}^{\prime} b & 0 & \tilde{b}^{\prime} \tilde{a} \\
0 & a^{\prime} b & 0 & a^{\prime} \tilde{a}
\end{array}\right)+\left(\begin{array}{cccc}
\tilde{b}^{\prime} b e^{-i 2\left(\omega_{3}^{\prime}-\omega_{3}\right) L} & 0 & \tilde{b}^{\prime} \tilde{a} e^{-i 2\left(\omega_{3}^{\prime}-\omega_{3}\right) L} & 0 \\
a^{\prime} b e^{-i 2\left(\omega_{3}^{\prime}-\omega_{3}\right) L} & 0 & a^{\prime} \tilde{a} e^{-i 2\left(\omega_{3}^{\prime}-\omega_{3}\right) L} & 0 \\
0 & \tilde{a}^{\prime} a e^{i 2\left(\omega_{3}^{\prime}-\omega_{3}\right) L} & 0 & -\tilde{a}^{\prime} \tilde{b} e^{i 2\left(\omega_{3}^{\prime}-\omega_{3}\right) L} \\
0 & -b^{\prime} a e^{i 2\left(\omega_{3}^{\prime}-\omega_{3}\right) L} & 0 & b^{\prime} b e^{i 2\left(\omega_{3}^{\prime}-\omega_{3}\right) L}
\end{array}\right)
$$

Secondly,

$$
\begin{aligned}
A_{0}(-L) \otimes^{\prime} C_{0}(-L)= & \left(\begin{array}{cc}
a \tilde{a}^{\prime} e^{-i\left(\omega_{3}-\omega_{3}^{\prime}\right) L}+\tilde{b} b^{\prime} e^{i\left(\omega_{3}-\omega_{3}^{\prime}\right) L} & a \tilde{b}^{\prime} e^{-i\left(\omega_{3}-\omega_{3}^{\prime}\right) L}-\tilde{b} a^{\prime} e^{i\left(\omega_{3}-\omega_{3}^{\prime}\right) L} \\
b a^{\prime} e^{-i\left(\omega_{3}-\omega_{3}^{\prime}\right) L}-\tilde{a} b^{\prime} e^{i\left(\omega_{3}-\omega_{3}^{\prime}\right) L} & b \tilde{b}^{\prime} e^{-i\left(\omega_{3}-\omega_{3}^{\prime}\right) L}+\tilde{a} a^{\prime} e^{i\left(\omega_{3}-\omega_{3}^{\prime}\right) L}
\end{array}\right) \\
& \otimes^{\prime}\left(\begin{array}{cc}
e^{-i\left(\omega_{3}^{\prime}-\omega_{3}\right) L} & 0 \\
0 & e^{i\left(\omega_{3}^{\prime}-\omega_{3}\right) L}
\end{array}\right)
\end{aligned}
$$


is equal to

$$
\left(\begin{array}{cccc}
a \tilde{a}^{\prime} & a \tilde{b}^{\prime} & 0 & 0 \\
0 & 0 & \tilde{b} b^{\prime} & -\tilde{b} a^{\prime} \\
b \tilde{a}^{\prime} & b \tilde{b}^{\prime} & 0 & 0 \\
0 & 0 & -\tilde{a} b^{\prime} & \tilde{a} a^{\prime}
\end{array}\right)+\left(\begin{array}{cccc}
\tilde{b} b^{\prime} e^{i 2\left(\omega_{3}-\omega_{3}^{\prime}\right) L} & -\tilde{b} a^{\prime} e^{i 2\left(\omega_{3}-\omega_{3}^{\prime}\right) L} & 0 & 0 \\
0 & 0 & a \tilde{a}^{\prime} e^{-i 2\left(\omega_{3}-\omega_{3}^{\prime}\right) L} & a \tilde{b}^{\prime} e^{-i 2\left(\omega_{3}-\omega_{3}^{\prime}\right) L} \\
-\tilde{a} b^{\prime} e^{i 2\left(\omega_{3}-\omega_{3}^{\prime}\right) L} & \tilde{a} a^{\prime} e^{i 2\left(\omega_{3}-\omega_{3}^{\prime}\right) L} & 0 & 0 \\
0 & 0 & b \tilde{a}^{\prime} e^{-i 2\left(\omega_{3}-\omega_{3}^{\prime}\right) L} & b \tilde{b}^{\prime} e^{-i 2\left(\omega_{3}-\omega_{3}^{\prime}\right) L}
\end{array}\right)
$$

Finally, combining (B13) and (B15), we have shown eq. (B33). 